\title{
New shape of the chirped bright, dark optical solitons and complex solutions for (2+1)-dimensional Ginzburg-Landau equation and modulation instability analysis
}

\author{
A. Houwe ${ }^{a, *}$, M. Inc ${ }^{b, c, d, \dagger}$, D. Baleanu ${ }^{e, f}$, H. Rezazadeh ${ }^{g}$, and S. Y. Doka ${ }^{h}$ \\ ${ }^{a}$ Department of Physics, Faculty of Science, the University of Maroua, P.O Box 814, Maroua, Cameroon. \\ *e-mail: ahouw220@yahoo.fr \\ ${ }^{b}$ Biruni University, Department of Computer Engineering, Istanbul, Turkey \\ ${ }^{c}$ Firat University, Science Faculty, Department of Mathematics, 23119 Elazig/Turkey. \\ ${ }^{d}$ Department of Medical Research, China Medical University, \\ Hospital, China Medical University, Taichung, Taiwan. \\ $\dagger$ e-mail: minc@firat.edu.tr, \\ ${ }^{e}$ Department of Mathematics, Cankaya University, Balgat 06530, Ankara, Turkey. \\ ${ }^{f}$ Institute of Space Sciences, Magurele-Bucharest R76900, Romania. \\ ${ }^{g}$ Faculty of Engineering Technology, Amol University of Special Modern Technologies, Amol, Iran. \\ ${ }^{h}$ Department of Physics, Faculty of Science, the University of Ngaoundere, P.O Box 454, Cameroon.
}

Received 1 December 2020; accepted 27 January 2021

\begin{abstract}
The investigation of the Ginzburg-Landau equation (GLE) has been done to find out and investigate new chirped bright, dark periodic and singular function solutions. For this purpose, we have used the traveling wave hypothesis and the chirp component. From there it was pointed out the constraint relation to the different arbitrary parameters of the GLE. Thereafter, we have employed the improved sub-ODE method to handle the nonlinear ordinary differential equation (NODE). In the paper, the virtue of the used analytical method has been highlighted via new chirped solitary waves. Besides, to emphasize the confrontation between the nonlinearity and dispersion terms, we have investigated the steady state of the newly obtained results. It has been obtained the Modulation instability (MI) gain spectra under the effect of the power incident and the transverse wave number. In our knowledge, these results are new compared to Refs. [28-34], and are going to be helpful to explain physical phenomena.
\end{abstract}

Keywords: Chirped bright and dark; Complex solutions; (2+1)-Ginzburg-Landau equation; modulation instability.

PACS: 42.81.Dp; 42.65.Tg

DOI: https://doi.org/10.31349/RevMexFis.67.040702

\section{Introduction}

The search for exact solutions of nonlinear systems has reached an unprecedented speed these days. The best known solitons solutions have found their applications in various fields just to name a few such as optical fibers, plasm, biology, quantum physics. Thus, solitons did not remain anonymous for a long time because of their direct implications in trans-continental and trans-oceanic data transport [1-24].

Without doubt, soliton is the one important wave which marveled in the field of data transport and securing it. It should also be noted that the most moving side of solitary waves comes from the fact that they are associated with chirped pulses. These chirped pulses, have been widely investigated in diverse shape in recent years by [27-29].

From this, many results in theoretical and experimentally have been followed with the mathematical tools to handle them $[10,35,36]$. These analytical methods facilitated the success of these results are among others, the Sine-Gordon expansion method, the modified $\exp (-\psi(\xi))$ expansion function method, $\left(\mathrm{G}^{\prime} / \mathrm{G}\right)$-expansion scheme, the trial expansion method, the new mapping method, the auxiliary equation method, the rational function method, and the
Riccati-Bernoulli sub-ODE method [25-30,35-45].

In this present work, an investigation will be carried out in order to formulate new shape of the chirped soliton solutions to the famous $(2+1)$-dimensional complex GinzburgLandau equation (GLE), of which skeletal structure is as follows [29-33]:

$$
i \psi_{t}+\frac{1}{2} \psi_{x x}+\frac{1}{2}(\alpha-i G) \psi_{y y}+(1-i \lambda)|\psi|^{2} \psi+i \gamma \psi=0,
$$

$\psi(x, t)$ represents complex wave profile on $(2+1)$ dimensional space time $R^{2+1}$, while $t$ represents the temporal variable and $x, y$ represent spatial variable. $\alpha, \lambda, \gamma$ and $G$ are real. The set of the CGLE Eq. (1) was recently used to depict the beginning of stationary periodic solutions in nonlinear stability problems. It takes the name of real Ginzburg-Landau equation when $(\alpha=\lambda=0)$ [30]. To get right to the purpose, the work is organized as follows: Section 2 is devoted to the traveling-wave solution. Section 3 is used the linear stability technic to study the modulation instability gain spectrum. Section 4 will depicts the obtained analytical results with their physical explanation. The last part of the work will present the conclusions of this work. 


\subsection{Analytical investigation and traveling waves solution}

The following envelope transformation is used to build soliton solution

$$
\psi(x, y, t)=\phi(\zeta) \exp [i(\xi+A(\zeta))]
$$

where $\phi(\zeta)$ is real function and the chirp component is $A(\zeta))$, with $\xi$ and $\zeta$ given by $\xi=k x+l y+v t+\xi_{0}$ and $\zeta=$ $n x+m y+\omega t+\eta_{0}$, respectively. Inserting Eq. (2) into Eq. (1) splits imaginary and real parts as

$$
\begin{aligned}
\left(n^{2}+\alpha m^{2}\right) \phi A & +2\left(n^{2}+\alpha m^{2}\right) \phi^{\prime} A^{\prime}+G m^{2} A^{\prime 2} \phi-G m^{2} \phi^{\prime \prime} \\
& +2(n k+\omega+\alpha l) \phi^{\prime}+2 G m l \phi A^{\prime}+\left(G l^{2}+2 \gamma\right) \phi-2 \lambda \phi^{3}=0,
\end{aligned}
$$

and

$$
\begin{aligned}
\left(n^{2}+\alpha m^{2}\right) \phi^{\prime \prime} & -\left(n^{2}+\alpha m^{2}\right) A^{\prime 2} \phi+2 G m^{2} A^{\prime} \phi^{\prime}+G m^{2} \phi A^{\prime \prime} \\
& -2(n k+\omega \alpha m l) A^{\prime} \phi+2 G m l \phi^{\prime}-\left(k^{2}+\alpha l^{2}+2 v\right) \phi+2 \phi^{3}=0,
\end{aligned}
$$

Suppose that the chirp is given by

$$
A(\zeta)=A_{0} \ln (|\phi(\zeta)|)
$$

where $A_{0}$ is an arbitrary constant to obtain later. Plugging Eq. (5) into Eq. (4) and Eq. (3), we get

$$
\ell_{1} \phi \phi^{\prime \prime}+\ell_{2} \phi^{2}+\ell_{3} \phi \phi^{\prime}+\ell_{4} \phi^{2}-2 \lambda \phi^{4}=0
$$

and

$$
\Lambda_{1} \phi \phi^{\prime \prime}-\Lambda_{2} \phi^{2}-\Lambda_{3} \phi \phi^{\prime}-\Lambda_{4} \phi^{2}+2 \phi^{4}=0
$$

where

$$
\begin{aligned}
& \ell_{1}=A_{0} n^{2}+A_{0} \alpha m^{2}-G m^{2}, \quad \ell_{2}=A_{0}\left(n^{2}+\alpha m^{2}+A_{0} G m^{2}\right), \ell_{3}=2\left(n k+\omega+\alpha m l+G m l A_{0}\right), \ell_{4}=G l^{2}+2 \gamma . \\
& \Lambda_{1}=n^{2}+\alpha m^{2}, \Lambda_{2}=A_{0}\left(A_{0} n^{2}+A_{0} \alpha m^{2}-G m^{2}\right), \Lambda_{3}=2\left(A_{0} n k+A_{0} \omega+A_{0} \alpha m l-G m l\right), \Lambda_{4}=k^{2}+\alpha l^{2}+2 v
\end{aligned}
$$

To deal with an analytical solutions to Eq. (7) and Eq. (6), we suppose that $\Lambda_{3}=\ell_{3}=0$. Consequently it is recovered

$$
A_{0}=\frac{G m l}{n k+\omega+\alpha m l} \text {. }
$$

Then Eq. (7) and Eq. (6) become

$$
\begin{aligned}
& \ell_{1} \phi \phi^{\prime \prime}+\ell_{2}{\phi^{\prime}}^{2}+\ell_{4} \phi^{2}-2 \lambda \phi^{4}=0, \\
& \Lambda_{1} \phi \phi^{\prime \prime}-\Lambda_{2}{\phi^{\prime}}^{2}-\Lambda_{4} \phi^{2}+2 \phi^{4}=0,
\end{aligned}
$$

It has become easy to investigate soliton-like solutions now. For this purpose, we considered the following expression as solution [45]

$$
\phi=\mu F^{n}(\zeta), \quad \mu>0
$$

and $n$ an arbitrary constant, while $F(\xi)$ is taken like solutions of the following ordinary differential equation [45]

$$
{F^{\prime}}^{2}(\zeta)=A F^{2-2 p}(\zeta)+B F^{2-p}(\zeta)+C F^{2}(\zeta)+D F^{2+p}(\zeta)+E F^{2+2 p}(\zeta) . \quad p>0
$$

With the homogeneous balance principle between $\phi \phi^{\prime \prime}$ and $\phi^{4}$, it is obtained $n+n+2 p=4 n \Rightarrow p=n$. From which Eq. (11) turns to

$$
\phi=\mu F^{p}(\zeta)
$$


Using the set of equations given by Eq. (13) together with Eq. (12) and make use of into Eq. (10) or Eq. (9) gives the set of system of equation in terms of $F^{j p}(\zeta)(j=2,3,4,5,6,8)$

$$
\begin{aligned}
-2 \lambda \mu^{4} F^{8 p}(\zeta) & +\left(6 \ell_{1} \mu^{2} p^{2} E+4 \ell_{2} \mu^{2} p^{2} E\right) F^{6 p}(\zeta)+\left(4 \ell_{2} \mu^{2} p^{2} D+5 \ell_{1} \mu^{2} p^{2} D\right) F^{5 p}(\zeta) \\
& +\left(4 \ell_{2} \mu^{2} p^{2} C+4 \ell_{1} \mu^{2} p^{2} C+\ell_{4} \mu^{2}\right) F^{4 p}(\zeta)+\left(4 \ell_{2} \mu^{2} p^{2} B+3 \ell_{1} \mu^{2} p^{2} B\right) F^{3 p}(\zeta) \\
& +\left(2 \ell_{1} \mu^{2} p^{2} A+4 \ell_{2} \mu^{2} p^{2} A\right) F^{2 p}(\zeta)=0 .
\end{aligned}
$$

Thereafter solving the obtained set of system of Eq. (14) by using the mathematical software Maple 18, it is revealed.

$$
A=0, B=0, C=-\frac{1}{4} \frac{\ell_{4}}{p^{2}\left(\ell_{2}+\ell_{1}\right)}, D=D, E=E .
$$

We can thus unroll the types of solutions of Eq. (1), as well as the corresponding chirped solutions

Case 1: If $A=0, B=0, D=0$, it is recovered bright soliton of Eq. (1):

$$
\begin{aligned}
\psi_{1,1}(x, y, t) & =e^{(i \xi)} \mu\left[\varepsilon \sqrt{-\frac{C}{E}} \operatorname{sech}(p \sqrt{C} \zeta)\right]^{\frac{1}{p}}\left[\left|\mu\left(\varepsilon \sqrt{-\frac{C}{E}} \operatorname{sech}(p \sqrt{C} \zeta)\right)^{\frac{1}{p}}\right|\right]^{\left(i A_{0}\right)}, \\
C & >0, \quad E<0, \quad \varepsilon \pm 1,
\end{aligned}
$$

and the bright chirp is

$$
A_{1,1}(x, y, t)=A_{0} \ln \left|\mu\left[\varepsilon \sqrt{-\frac{C}{E}} \operatorname{sech}(p \sqrt{C} \zeta)\right]^{\frac{1}{p}}\right|, \quad C>0, \quad E<0, \quad \varepsilon \pm 1,
$$

a periodic function solutions

$$
\begin{gathered}
\psi_{1,2}(x, y, t)=e^{(i \xi)} \mu\left[\varepsilon \sqrt{-\frac{C}{E}} \sec (p \sqrt{-C} \zeta)\right]^{\frac{1}{p}}\left[\left|\mu\left(\varepsilon \sqrt{-\frac{C}{E}} \sec (p \sqrt{-C} \zeta)\right)^{\frac{1}{p}}\right|\right]^{\left(i A_{0}\right)}, \\
C<0, \quad E>0, \quad \varepsilon \pm 1,
\end{gathered}
$$

and the periodic chirp

$$
A_{1,2}(x, y, t)=A_{0} \ln \left|\left[\varepsilon \sqrt{-\frac{C}{E}} \sec (p \sqrt{-C} \zeta)\right]^{\frac{1}{p}}\right|, \quad C<0, \quad E>0, \quad \varepsilon \pm 1,
$$

then a rational solution

$$
\psi_{1,3}(x, y, t)=e^{(i \xi)} \mu\left[\frac{\varepsilon}{p \sqrt{E} \zeta}\right]^{\frac{1}{p}}\left[\mid \mu\left(\frac{\varepsilon}{p \sqrt{E} \zeta}\right)^{\frac{1}{p}}\right]^{\left(i A_{0}\right)}, \quad C=0, \quad E>0, \quad \varepsilon \pm 1 .
$$

and the rational chirp

$$
A_{1,3}(x, y, t)=A_{0} \ln \left|\mu\left[\frac{\varepsilon}{p \sqrt{E} \zeta}\right]^{\frac{1}{p}}\right|, \quad C=0, \quad E>0, \quad \varepsilon \pm 1 .
$$

Case 2: By setting the variables $A=0, B=0$, we deduce three forms of solutions of Eq. (5):

$$
\begin{gathered}
\psi_{2,1}(x, y, t)=e^{(i \xi)} \mu\left[\frac{1}{\cosh (p \sqrt{C} \zeta)-\frac{D}{2 C}}\right]^{\frac{1}{p}}\left[\left|\mu\left(\frac{1}{\cosh (p \sqrt{C} \zeta)-\frac{D}{2 C}}\right)^{\frac{1}{p}}\right|\right]^{\left(i A_{0}\right)}, \\
C>0, D<2 C, \quad E=\frac{D^{2}}{4 C}-C
\end{gathered}
$$


and the chirp

$$
A_{2,1}(x, y, t)=A_{0} \ln \left|\mu\left[\frac{1}{\cosh (p \sqrt{C} \zeta)-\frac{D}{2 C}}\right]^{\frac{1}{p}}\right|, \quad C>0, D<2 C, \quad E=\frac{D^{2}}{4 C}-C,
$$

it is gained for $C>0, E>0, D=-2 \sqrt{C E}, \quad \varepsilon= \pm 1$,

$$
\psi_{2,2}(x, y, t)=e^{(i \xi)} \mu\left[\frac{1}{2} \sqrt{\frac{C}{E}}\left(1+\varepsilon \tanh \left(\frac{p}{2} \sqrt{C} \zeta\right)\right)\right]^{\frac{1}{p}}\left[\left|\mu\left(\frac{1}{2} \sqrt{\frac{C}{E}}\left(1+\varepsilon \tanh \left(\frac{p}{2} \sqrt{C} \zeta\right)\right)\right)^{\frac{1}{p}}\right|\right]^{\left(i A_{0}\right)},
$$

the corresponding chirp gives

$$
A_{2,2}(x, y, t)=A_{0} \ln \left|\mu\left[\frac{1}{2} \sqrt{\frac{C}{E}}\left(1+\varepsilon \tanh \left(\frac{p}{2} \sqrt{C} \zeta\right)\right)\right]^{\frac{1}{p}}\right|, \quad C>0, \quad E>0, \quad D=-2 \sqrt{C E}, \quad \varepsilon= \pm 1
$$

and

$$
\psi_{2,3}(x, y, t)=e^{(i \xi)} \mu\left[\frac{4 D}{(p D \zeta)^{2}-4 E}\right]^{\frac{1}{p}}\left[\left|\mu\left(\frac{4 D}{(p D \zeta)^{2}-4 E}\right)^{\frac{1}{p}}\right|\right]^{\left(i A_{0}\right)}, \quad C=0, E<0 .
$$

with the chirp

$$
A_{2,3}(x, y, t)=A_{0}\left|\mu\left[\frac{4 D}{(p D \zeta)^{2}-4 E}\right]^{\frac{1}{p}}\right|, \quad C=0, E<0 .
$$

Case 3: Considering $A=B=0, C>0$, we have gained combined bright soliton and hyperbolic functions solutions of Eq. (5):

$$
\begin{aligned}
\psi_{3,1}(x, y, t) & =e^{(i \xi)} \mu\left[\frac{2 C \operatorname{sech}^{2}\left(\frac{p}{2} \sqrt{C} \zeta\right)}{2 \sqrt{D^{2}-4 C E}-\left(\sqrt{D^{2}-4 C E}+D\right) \operatorname{sech}^{2}\left(\frac{p}{2} \sqrt{C} \zeta\right)}\right]^{\frac{1}{p}} \\
& \times\left[\mid \mu\left(\frac{2 C \operatorname{sech}^{2}\left(\frac{p}{2} \sqrt{C} \zeta\right)}{2 \sqrt{D^{2}-4 C E}-\left(\sqrt{D^{2}-4 C E}+D\right) \operatorname{sech}^{2}\left(\frac{p}{2} \sqrt{C} \zeta\right)}\right)^{\frac{1}{p}}\right]^{\left(i A_{0}\right)} D^{2}-4 C E>0,
\end{aligned}
$$

the corresponding chirp

$$
\begin{aligned}
A_{3,1}(x, y, t) & =A_{0} \ln \left|\left[\frac{2 C \operatorname{sech}^{2}\left(\frac{p}{2} \sqrt{C} \zeta\right)}{2 \sqrt{D^{2}-4 C E}-\left(\sqrt{D^{2}-4 C E}+D\right) \operatorname{sech}^{2}\left(\frac{p}{2} \sqrt{C} \zeta\right)}\right]^{\frac{1}{p}}\right|, \quad D^{2}-4 C E>0, \\
\psi_{3,2}(x, y, t) & =e^{(i \xi)} \mu\left[\frac{2 C \operatorname{csch}^{2}\left(\frac{p}{2} \sqrt{C} \zeta\right)}{2 \sqrt{D^{2}-4 C E}+\left(\sqrt{D^{2}-4 C E}-D\right) \operatorname{csch}^{2}\left(\frac{p}{2} \sqrt{C} \zeta\right)}\right]^{\frac{1}{p}} \\
& \times\left[\mu\left(\frac{2 C \operatorname{csch}^{2}\left(\frac{p}{2} \sqrt{C} \zeta\right)}{2 \sqrt{D^{2}-4 C E}+\left(\sqrt{D^{2}-4 C E}-D\right) \operatorname{csch}^{2}\left(\frac{p}{2} \sqrt{C} \zeta\right)}\right)^{\frac{1}{p}} \mid\right]^{\left(i A_{0}\right)}, \quad D^{2}-4 C E>0,
\end{aligned}
$$

with the corresponding chirp

$$
A_{3,2}(x, y, t)=A_{0} \ln \left|\mu\left[\frac{2 C \operatorname{csch}^{2}\left(\frac{p}{2} \sqrt{C} \zeta\right)}{2 \sqrt{D^{2}-4 C E}+\left(\sqrt{D^{2}-4 C E}-D\right) \operatorname{csch}^{2}\left(\frac{p}{2} \sqrt{C} \zeta\right)}\right]^{\frac{1}{p}}\right|, \quad D^{2}-4 C E>0,
$$

for $D^{2}-4 C E>0, \varepsilon= \pm 1$

$$
\psi_{3,3}(x, y, t)=e^{(i \xi)} \mu\left[\frac{2 C}{\varepsilon \sqrt{D^{2}-4 C E} \cosh (p \sqrt{C} \xi)-D}\right]^{\frac{1}{p}}\left[\left|\mu\left(\frac{2 C}{\varepsilon \sqrt{D^{2}-4 C E} \cosh (p \sqrt{C} \zeta)-D}\right)^{\frac{1}{p}}\right|\right]^{\left(i A_{0}\right)},
$$


the chirp gives

$$
A_{3,3}(x, y, t)=A_{0} \ln \left|\mu\left[\frac{2 C}{\varepsilon \sqrt{D^{2}-4 C E} \cosh (p \sqrt{C} \zeta)-D}\right]^{\frac{1}{p}}\right|, \quad D^{2}-4 C E>0, \quad \varepsilon= \pm 1
$$

for $D^{2}-4 C E<0, \varepsilon= \pm 1$

$$
\begin{aligned}
\psi_{3,4}(x, y, t) & =e^{(i \xi)} \mu\left[\frac{2 C}{\varepsilon \sqrt{-\left(D^{2}-4 C E\right)} \sinh (p \sqrt{C} \zeta)-D}\right]^{\frac{1}{p}} \\
& \times\left[\left|\mu\left(\frac{2 C}{\varepsilon \sqrt{-\left(D^{2}-4 C E\right)} \sinh (p \sqrt{C} \zeta)-D}\right)^{\frac{1}{p}}\right|\right]^{\left(i A_{0}\right)}
\end{aligned}
$$

the chirp is

$$
\begin{gathered}
A_{3,4}(x, y, t)=A_{0} \ln \left|\mu\left[\frac{2 C}{\varepsilon \sqrt{-\left(D^{2}-4 C E\right)} \sinh (p \sqrt{C} \zeta)-D}\right]^{\frac{1}{p}}\right|, \quad D^{2}-4 C E<0, \quad \varepsilon= \pm 1 \\
\psi_{3,5}(x, y, t)=e^{(i \xi)} \mu\left[-\frac{C}{D}\left(1+\varepsilon \tanh \left(\frac{p}{2} \sqrt{C} \zeta\right)\right]^{\frac{1}{p}}\left[\mid \mu\left(-\frac{C}{D}\left(1+\varepsilon \tanh \left(\frac{p}{2} \sqrt{C} \zeta\right)\right)^{\frac{1}{p}} \mid\right]^{\left(i A_{0}\right)}\right.\right. \\
D^{2}-4 C E=0, \quad \varepsilon= \pm 1
\end{gathered}
$$

the chirp is given by

$$
A_{3,5}(x, y, t)=A_{0} \ln \left|\mu\left[-\frac{C}{D}\left(1+\varepsilon \tanh \left[\frac{p}{2} \sqrt{C} \zeta\right]\right)\right]^{\frac{1}{p}}\right|, \quad D^{2}-4 C E=0, \quad \varepsilon= \pm 1
$$

for $D^{2}-4 C E=0, \varepsilon= \pm 1$

$$
\psi_{3,6}(x, y, t)=e^{(i \xi)} \mu\left[-\frac{C}{D}\left(1+\varepsilon \operatorname{coth}\left(\frac{p}{2} \sqrt{C} \zeta\right)\right]^{\frac{1}{p}}\left[\left|\mu\left(-\frac{C}{D}\left(1+\varepsilon \operatorname{coth}\left[\frac{p}{2} \sqrt{C} \zeta\right]\right]\right)^{\frac{1}{p}}\right|\right]^{\left(i A_{0}\right)}\right.
$$

the corresponding chirp

$$
A_{3,6}(x, y, t)=A_{0} \ln \left|\mu\left[-\frac{C}{D}\left(1+\varepsilon \operatorname{coth}\left[\frac{p}{2} \sqrt{C} \zeta\right]\right)\right]^{\frac{1}{p}}\right|, \quad D^{2}-4 C E=0, \quad \varepsilon= \pm 1
$$

for $E>0, \quad \varepsilon= \pm 1$

$$
\psi_{3,7}(x, y, t)=e^{(i \xi)} \mu\left[-\frac{C \operatorname{sech}^{2}\left(\frac{p}{2} \sqrt{C} \zeta\right)}{D+2 \varepsilon \sqrt{C E} \tanh \left(\frac{p}{2} \sqrt{C} \zeta\right)}\right]^{\frac{1}{p}}\left[\left|\mu\left(-\frac{C \operatorname{sech}^{2}\left(\frac{p}{2} \sqrt{C} \zeta\right)}{D+2 \varepsilon \sqrt{C E} \tanh \left(\frac{p}{2} \sqrt{C} \zeta\right)}\right)^{\frac{1}{p}}\right|\right]^{\left(i A_{0}\right)},
$$

the chirp is revealed as

$$
A_{3,7}(x, y, t)=A_{0} \ln \left|\mu\left[-\frac{C \operatorname{sech}^{2}\left(\frac{p}{2} \sqrt{C} \zeta\right)}{D+2 \varepsilon \sqrt{C E} \tanh \left(\frac{p}{2} \sqrt{C} \zeta\right)}\right]^{\frac{1}{p}}\right|, \quad E>0, \quad \varepsilon= \pm 1
$$

for $E>0, \quad \varepsilon= \pm 1$

$$
\psi_{3,8}(x, y, t)=e^{(i \xi)} \mu\left[\frac{C \operatorname{csch}^{2}\left(\frac{p}{2} \sqrt{C} \zeta\right)}{D+2 \varepsilon \sqrt{C E} \operatorname{coth}\left(\frac{p}{2} \sqrt{C} \zeta\right)}\right]^{\frac{1}{p}}\left[\left|\mu\left(\frac{C \operatorname{csch}^{2}\left(\frac{p}{2} \sqrt{C} \zeta\right)}{D+2 \varepsilon \sqrt{C E} \operatorname{coth}\left(\frac{p}{2} \sqrt{C} \zeta\right)^{\frac{1}{2}}}\right)\right|\right]^{\left(i A_{0}\right)},
$$


and the corresponding chirp

$$
\begin{aligned}
A_{3,8}(x, y, t) & =A_{0} \ln \left|\mu\left[\frac{C \operatorname{csch}^{2}\left(\frac{p}{2} \sqrt{C} \zeta\right)}{D+2 \varepsilon \sqrt{C E} \operatorname{coth}\left(\frac{p}{2} \sqrt{C} \zeta\right)}\right]^{\frac{1}{p}}\right|, \quad E>0, \quad \varepsilon= \pm 1 \\
\psi_{3,9}(x, y, t) & =e^{(i \xi)} \mu\left[\frac{-C D \operatorname{sech}^{2}\left(\frac{p}{2} \sqrt{C} \zeta\right)}{D^{2}-C E\left(1+\varepsilon \tanh \left(\frac{p}{2} \sqrt{C} \zeta\right)\right)^{2}}\right]^{\frac{1}{2}} \\
& \times\left[\mu\left(\frac{-C D \operatorname{sech}^{2}\left(\frac{p}{2} \sqrt{C} \zeta\right)}{D^{2}-C E\left(1+\varepsilon \tanh \left(\frac{p}{2} \sqrt{C} \zeta\right)\right)^{2}}\right)^{\frac{1}{P}} \mid\right]^{\left(i A_{0}\right)},
\end{aligned}
$$

the chirp gives

$$
\begin{aligned}
A_{3,9}(x, y, t) & =A_{0} \ln \left|\mu\left[\frac{-C D \operatorname{sech}^{2}\left(\frac{p}{2} \sqrt{C} \zeta\right)}{D^{2}-C E\left(1+\varepsilon \tanh \left(\frac{p}{2} \sqrt{C} \zeta\right)\right)^{2}}\right]^{\frac{1}{P}}\right| \\
\psi_{3,10}(x, y, t) & =e^{(i \xi)} \mu\left[\frac{C D \operatorname{csch}^{2}\left(\frac{p}{2} \sqrt{C} \zeta\right)}{D^{2}-C E\left(1+\varepsilon \operatorname{coth}\left(\frac{p}{2} \sqrt{C} \zeta\right)\right)^{2}}\right]^{\frac{1}{P}} \\
& \left.\times\left[\mu\left(\frac{C D \operatorname{csch}^{2}\left(\frac{p}{2} \sqrt{C} \zeta\right)}{D^{2}-C E\left(1+\varepsilon \operatorname{coth}\left(\frac{p}{2} \sqrt{C} \zeta\right)\right)^{2}}\right)^{\frac{1}{p}}\right]\right]^{\left(i A_{0}\right)} .
\end{aligned}
$$

and the corresponding chirp as

$$
A_{3,11}(x, y, t)=A_{0} \ln \left|\mu\left[\frac{C D \operatorname{csch}^{2}\left(\frac{p}{2} \sqrt{C} \zeta\right)}{D^{2}-C E\left(1+\varepsilon \operatorname{coth}\left(\frac{p}{2} \sqrt{C} \zeta\right)\right)^{2}}\right]^{\frac{1}{p}}\right| .
$$

Case 4: Considering $A=B=0, C<0$, we have gained combined bright soliton and hyperbolic functions as solutions For $D^{2}-4 C E>0$

$$
\begin{aligned}
\psi_{4,1}(x, y, t) & =e^{(i \xi)}\left[\frac{-2 C \sec ^{2}\left(\frac{p}{2} \sqrt{-C} \zeta\right)}{2 \sqrt{D^{2}-4 C E}-\left(\sqrt{D^{2}-4 C E}-D\right) \sec ^{2}\left(\frac{p}{2} \sqrt{-C} \zeta\right)}\right]^{\frac{1}{p}} \\
& \times\left[\mid \mu\left(\frac{-2 C \sec ^{2}\left(\frac{p}{2} \sqrt{-C} \zeta\right)}{2 \sqrt{D^{2}-4 C E}-\left(\sqrt{D^{2}-4 C E}-D\right) \sec ^{2}\left(\frac{p}{2} \sqrt{-C} \zeta\right)}\right)^{\frac{1}{p}}\right]^{\left(i A_{0}\right)},
\end{aligned}
$$

the chirp gives

$$
A_{4,2}(x, y, t)=A_{0} \ln \left|\mu\left[\frac{-2 C \sec ^{2}\left(\frac{p}{2} \sqrt{-C} \zeta\right)}{2 \sqrt{D^{2}-4 C E}-\left(\sqrt{D^{2}-4 C E}-D\right) \sec ^{2}\left(\frac{p}{2} \sqrt{-C} \zeta\right)}\right]^{\frac{1}{p}}\right|, \quad D^{2}-4 C E>0,
$$


for $D^{2}-4 C E>0$,

$$
\begin{aligned}
\psi_{4,3}(x, y, t) & =e^{(i \xi)} \mu\left[\frac{2 C \csc ^{2}\left(\frac{p}{2} \sqrt{-C} \zeta\right)}{2 \sqrt{D^{2}-4 C E}-\left(\sqrt{D^{2}-4 C E}+D\right) \csc ^{2}\left(\frac{p}{2} \sqrt{-C} \zeta\right)}\right]^{\frac{1}{p}} \\
& \times\left[\mid \mu\left(\frac{2 C \csc ^{2}\left(\frac{p}{2} \sqrt{-C} \zeta\right)}{2 \sqrt{D^{2}-4 C E}-\left(\sqrt{D^{2}-4 C E}+D\right) \csc ^{2}\left(\frac{p}{2} \sqrt{-C} \zeta\right)}\right)^{\frac{1}{p}}\right]^{\left(i A_{0}\right)},
\end{aligned}
$$

the chirp gives

$$
A_{4,4}(x, y, t)=A_{0} \ln \left|\mu\left[\frac{2 C \csc ^{2}\left(\frac{p}{2} \sqrt{-C} \zeta\right)}{2 \sqrt{D^{2}-4 C E}-\left(\sqrt{D^{2}-4 C E}+D\right) \csc ^{2}\left(\frac{p}{2} \sqrt{-C} \zeta\right)}\right]^{\frac{1}{p}}\right|, \quad D^{2}-4 C E>0,
$$

for $D^{2}-4 C E>0, \varepsilon= \pm 1$,

$$
\begin{aligned}
\psi_{4,5}(x, y, t) & =e^{(i \xi)} \mu\left[\frac{2 C \sec (p \sqrt{-C} \zeta)}{\varepsilon \sqrt{D^{2}-4 C E}-D \sec (p \sqrt{-C} \zeta)}\right]^{\frac{1}{p}} \\
& \times\left[\left|\mu\left(\frac{2 C \sec (p \sqrt{-C} \zeta)}{\varepsilon \sqrt{D^{2}-4 C E}-D \sec (p \sqrt{-C} \zeta)}\right)^{\frac{1}{p}}\right|\right]^{\left(i A_{0}\right)},
\end{aligned}
$$

the chirp reads as follows

$$
A_{4,5}(x, y, t)=A_{0} \ln \left|\mu\left[\frac{2 C \sec (p \sqrt{-C} \zeta)}{\varepsilon \sqrt{D^{2}-4 C E}-D \sec (p \sqrt{-C} \zeta)}\right]^{\frac{1}{p}}\right|, \quad D^{2}-4 C E>0, \quad \varepsilon= \pm 1,
$$

for $D^{2}-4 C E>0, \varepsilon= \pm 1$

$$
\psi_{4,6}(x, y, t)=e^{(i \xi)} \mu\left[\frac{2 C \csc (p \sqrt{-C} \zeta)}{\varepsilon \sqrt{D^{2}-4 C E}-D \csc (p \sqrt{-C} \zeta)}\right]^{\frac{1}{p}}\left[\left|\mu\left(\frac{2 C \csc (p \sqrt{-C} \zeta)}{\varepsilon \sqrt{D^{2}-4 C E}-D \csc (p \sqrt{-C} \zeta)}\right)^{\frac{1}{p}}\right|\right]^{\left(i A_{0}\right)},
$$

the chirp is given by

$$
A_{4,6}(x, y, t)=A_{0} \ln \left|\mu\left[\frac{2 C \csc (p \sqrt{-C} \zeta)}{\varepsilon \sqrt{D^{2}-4 C E}-D \csc (p \sqrt{-C} \zeta)}\right]^{\frac{1}{p}}\right|, \quad D^{2}-4 C E>0, \quad \varepsilon= \pm 1,
$$

for $E>0, \varepsilon= \pm 1$,

$$
\psi_{4,7}(x, y, t)=e^{(i \xi)} \mu\left[-\frac{C \sec ^{2}\left(\frac{p}{2} \sqrt{-C} \zeta\right)}{D+2 \varepsilon \sqrt{-C E} \tan \left(\frac{p}{2} \sqrt{-C} \zeta\right)}\right]^{\frac{1}{p}}\left[\left|\mu\left(-\frac{C \sec ^{2}\left(\frac{p}{2} \sqrt{-C} \zeta\right)}{D+2 \varepsilon \sqrt{-C E} \tan \left(\frac{p}{2} \sqrt{-C} \zeta\right)}\right)^{\frac{1}{p}}\right|\right]^{\left(i A_{0}\right)},
$$

the chirp is obtained

$$
A_{4,7}(x, y, t)=A_{0} \ln \left|\mu\left[-\frac{C \sec ^{2}\left(\frac{p}{2} \sqrt{-C} \zeta\right)}{D+2 \varepsilon \sqrt{-C E} \tan \left(\frac{p}{2} \sqrt{-C} \zeta\right)}\right]^{\frac{1}{p}}\right|, \quad E>0, \quad \varepsilon= \pm 1,
$$

for $D^{2}-4 C E>0, E>0, \varepsilon= \pm 1$

$$
\psi_{4,8}(x, y, t)=e^{(i \xi)} \mu\left[-\frac{C \csc ^{2}\left(\frac{p}{2} \sqrt{-C} \zeta\right)}{D+2 \varepsilon \sqrt{-C E} \cot \left(\frac{p}{2} \sqrt{-C} \zeta\right)}\right]^{\frac{1}{p}}\left[\left|\mu\left(-\frac{C \csc ^{2}\left(\frac{p}{2} \sqrt{-C} \zeta\right)}{D+2 \varepsilon \sqrt{-C E} \cot \left(\frac{p}{2} \sqrt{-C} \zeta\right)}\right)^{\frac{1}{p}}\right|\right]^{\left(i A_{0}\right)},
$$

the chirp is

$$
A_{4,8}(x, y, t)=A_{0} \ln \left|\mu\left[-\frac{C \csc ^{2}\left(\frac{p}{2} \sqrt{-C} \zeta\right)}{D+2 \varepsilon \sqrt{-C E} \cot \left(\frac{p}{2} \sqrt{-C} \zeta\right)}\right]^{\frac{1}{p}}\right|, \quad D^{2}-4 C E>0, E>0, \quad \varepsilon= \pm 1 .
$$


Case 5: For $A=B=0, \quad C>0, \quad \varepsilon= \pm 1$,

$$
\psi_{5,1}(x, y, t)=e^{(i \xi)} \mu\left[\frac{4 C p^{2} e^{(p \varepsilon \sqrt{C} \zeta)}}{\left(e^{\varepsilon p \sqrt{C} \zeta}-D p^{2}\right)^{2}-4 C E p^{4}}\right]^{\frac{1}{p}}\left[\left|\mu\left(\frac{4 C p^{2} e^{(p \varepsilon \sqrt{C \zeta})}}{\left(e^{\varepsilon p \sqrt{C \zeta}}-D p^{2}\right)^{2}-4 C E p^{4}}\right)^{\frac{1}{p}}\right|\right]^{\left(i A_{0}\right)},
$$

the chirp component is

$$
\begin{gathered}
A_{5,1}(x, y, t)=A_{0} \ln \left|\mu\left[\frac{4 C p^{2} e^{(p \varepsilon \sqrt{C} \xi)}}{\left(e^{\varepsilon p \sqrt{C} \zeta}-D p^{2}\right)^{2}-4 C E p^{4}}\right]^{\frac{1}{p}}\right|, \quad C>0, \quad \varepsilon= \pm 1, \\
\psi_{5,2}(x, y, t)=e^{(i \xi)} \mu\left[\frac{4 C p^{2} e^{(p \varepsilon \sqrt{C} \zeta)}}{-1+4 C E p^{4} e^{2 \varepsilon p \sqrt{C \zeta}}}\right]^{\frac{1}{p}}\left[\left|\mu\left(\frac{4 C p^{2} e^{(p \varepsilon \sqrt{C \zeta})}}{-1+4 C E p^{4} e^{2 \varepsilon p \sqrt{C \zeta}}}\right)^{\frac{1}{p}}\right|\right]^{\left(i A_{0}\right)}, \\
C>0, D=0, \quad \varepsilon= \pm 1,
\end{gathered}
$$

the chirp gives

$$
\begin{aligned}
& A_{5,2}(x, y, t)=A_{0} \ln \left|\mu\left[\frac{4 C p^{2} e^{(p \varepsilon \sqrt{C} \zeta)}}{-1+4 C E p^{4} e^{2 \varepsilon p \sqrt{C} \zeta}}\right]^{\frac{1}{p}}\right|, \quad C>0, D=0, \quad \varepsilon= \pm 1, \\
& \psi_{5,3}(x, y, t)=e^{(i \xi)} \mu\left[\frac{\varepsilon}{p \sqrt{E} \zeta}\right]^{\frac{1}{p}}\left[\left|\mu\left(\frac{\varepsilon}{p \sqrt{E} \zeta}\right)^{\frac{1}{p}}\right|\right]^{\left(i A_{0}\right)}, \quad E>0, C=D=0, \quad \varepsilon= \pm 1 .
\end{aligned}
$$

The last chirp gives

$$
A_{5,3}(x, y, t)=A_{0} \ln \left|\mu\left[\frac{\varepsilon}{p \sqrt{E} \zeta}\right]^{\frac{1}{p}}\right|, \quad E>0, C=D=0, \quad \varepsilon= \pm 1 .
$$

\subsection{Modulation analysis}

This section will be using linear analysis technique to take out the modulation instability (MI) gain spectrum. Assuming the steady state solution of Eq. (1) in the form of:

$$
\psi(x, y, t)=\left[\sqrt{P_{0}}+B(x, y, t)\right] e^{i \phi_{N L}}, \quad \phi_{N L}=\delta P_{0} x
$$

where $P_{0}$ is the incident power. $B(x, y, t)$ is the small perturbation component and $B^{*}(x, y, t)$ is the complex conjugate. Inserting Eq. (66) into Eq. (1) lead to

$$
\psi(x, y, t)=i B_{t}+\frac{1}{2} B_{x x}+\frac{1}{2}(1-\alpha G) B_{y y}+(1-i \lambda)\left(P_{0}\left(2 B+B^{*}\right)+i \gamma B=0 .\right.
$$

Suppose the solution of Eq. (67) is in the following expression

$$
B(x, y, t)=a_{1} e^{i[K x+\Gamma y-\Omega t]}+a_{2} e^{-i[K x+\Gamma y-\Omega t]} .
$$

where $a_{j}(j=1,2)$ are reals, and $K$ and $\Omega$ are wave numbers and the modulation frequency, respectively. The quantity $\Gamma$ is the transverse wave number of the perturbation. Inserting Eq. (68) into Eq. (67) gives the set of linear of coupled equations for $a_{1}$ and $a_{2}$

$$
\begin{gathered}
\left(\Omega-2 i \lambda P_{0}-\frac{1}{2} K^{2}+2 P_{0}-\frac{1}{2} \alpha \Gamma^{2}+\lambda \gamma+\frac{1}{2} i G \Gamma^{2}+i \gamma\right) a_{1}+\left(P_{0}-i \lambda P_{0}\right) a_{2}=0 \\
\left(P_{0}-i \lambda P_{0}\right) a_{1}+\left(-\frac{1}{2} K^{2}+2 P_{0}-\Omega-2 i \lambda P_{0}+\frac{1}{2} i G \Gamma^{2}+i \gamma-\frac{1}{2} \alpha \Gamma^{2}+\lambda \gamma\right) a_{2}=0 .
\end{gathered}
$$


This set of coupled of equation has a nontrivial solution when the determinant of the matrix below vanishes

$$
\left[\begin{array}{cc}
\Omega-2 i \lambda P_{0}-\frac{1}{2} K^{2}+2 P_{0}-\frac{1}{2} \alpha \Gamma^{2}+\lambda \gamma+\frac{1}{2} i G \Gamma^{2}+i \gamma & P_{0}-i \lambda P_{0} \\
P_{0}-i \lambda P_{0} & -\frac{1}{2} K^{2}+2 P_{0}-\Omega-2 i \lambda P_{0}+\frac{1}{2} i G \Gamma^{2}+i \gamma-\frac{1}{2} \alpha \Gamma^{2}+\lambda \gamma
\end{array}\right]
$$

Thereafter, the MI gain spectrum is revealed as

$$
\begin{aligned}
2 \operatorname{Im}(K) & =\left(-4 i K^{2} \gamma-4 G \Gamma^{2} \gamma-24 i \lambda P_{0}^{2}-8 P_{0} \alpha \Gamma^{2}+16 i P_{0} \gamma+8 i \lambda \gamma^{2}-4 K^{2} \lambda \gamma+4 \lambda^{2} \gamma^{2}-G^{2} \Gamma^{4}-12 \lambda^{2} P_{0}^{2}\right. \\
& -8 K^{2} P_{0}+12 P_{0}^{2}+\alpha^{2} \Gamma^{4}+8 i \lambda P_{0} \alpha \Gamma^{2}+4 i \lambda \gamma G \Gamma^{2}+2 K^{2} \alpha \Gamma^{2}+32 P_{0} \lambda \gamma+8 \lambda P_{0} G \Gamma^{2}+8 i \lambda P_{0} K^{2} \\
& \left.-2 K^{2} i G \Gamma^{2}+8 P_{0} i G \Gamma^{2}-2 i \alpha \Gamma^{4} G-16 i \lambda^{2} P_{0} \gamma-4 i \alpha \Gamma^{2} \gamma-4 \alpha \Gamma^{2} \lambda \gamma-4 \gamma^{2}+K^{4}\right)^{\frac{1}{2}}
\end{aligned}
$$

Now to investigate the behavior of Eq. (71), we first point out the condition of the obtaining the steady state. So, it is important to highlight the fact that the perturbation grow exponentially, when the wave number value contains the imaginary part, in this condition the steady state solution is unstable. However, in case of small perturbation and having the wave number with real value, the steady state of the solution is stable.

Here two cases are going to be discussed:

- Case 1: In this case, the steady state is stable again and a small perturbution is seen if $K$ is real

$$
\begin{aligned}
& \left(-4 i K^{2} \gamma-4 G \Gamma^{2} \gamma-24 i \lambda P_{0}^{2}-8 P_{0} \alpha \Gamma^{2}+16 i P_{0} \gamma+8 i \lambda \gamma^{2}-4 K^{2} \lambda \gamma+4 \lambda^{2} \gamma^{2}-G^{2} \Gamma^{4}-12 \lambda^{2} P_{0}^{2}-8 K^{2} P_{0}\right. \\
& \quad+12 P_{0}^{2}+\alpha^{2} \Gamma^{4}+8 i \lambda P_{0} \alpha \Gamma^{2}+4 i \lambda \gamma G \Gamma^{2}+2 K^{2} \alpha \Gamma^{2}+32 P_{0} \lambda \gamma+8 \lambda P_{0} G \Gamma^{2}+8 i \lambda P_{0} K^{2}-2 K^{2} i G \Gamma^{2}+8 P_{0} i G \Gamma^{2} \\
& \left.\quad-2 i \alpha \Gamma^{4} G-16 i \lambda^{2} P_{0} \gamma-4 i \alpha \Gamma^{2} \gamma-4 \alpha \Gamma^{2} \lambda \gamma-4 \gamma^{2}+K^{4}\right)^{\frac{1}{2}}>0
\end{aligned}
$$

but if $\lambda, \gamma$ and $G$ are non zero value, the inequality Eq. (72) is invalid. Consequently the steady is still unstable.

a)

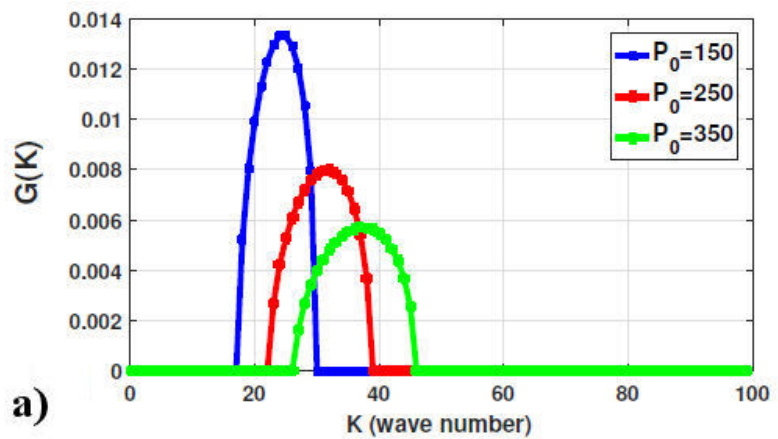

c)

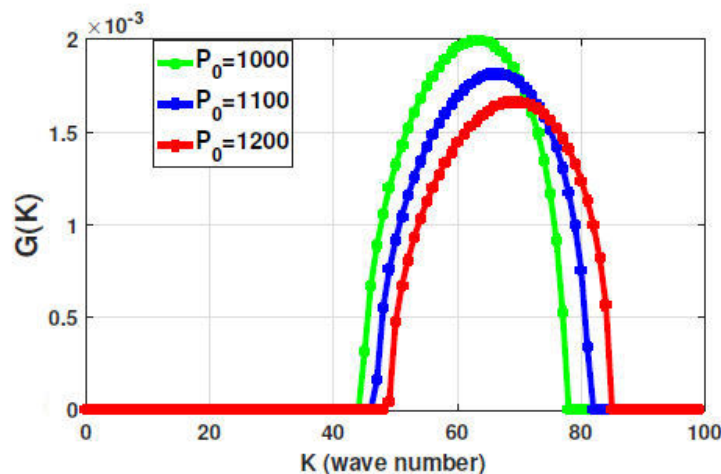

b)
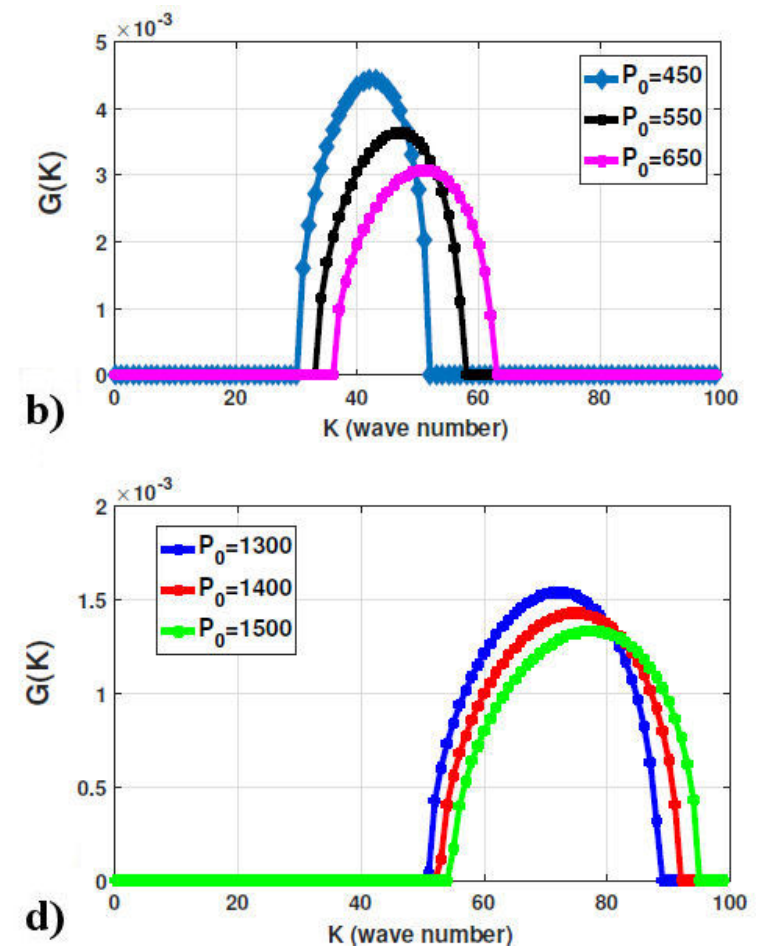

FIGURE 1. (Color online) Variation of MI gain spectrum versus wave number with the effect of the incident power a) $\left[P_{0}=150, P_{0}=\right.$ $\left.250, P_{0}=350\right]$, b) $\left[P_{0}=450, P_{0}=550, P_{0}=650\right]$, c) $\left[P_{0}=1000, P_{0}=1100, P_{0}=1200\right]$, d) $\left[P_{0}=1300, P_{0}=1400, P_{0}=\right.$ 1500] at $\alpha=0.1, \Gamma=0.5$. 
- Case 2: In this case, the MI occurs when $K$ is imaginary due to the fact that the perturbation increases exponentially and in the same time

$$
\begin{aligned}
& \left(-4 i K^{2} \gamma-4 G \Gamma^{2} \gamma-24 i \lambda P_{0}^{2}-8 P_{0} \alpha \Gamma^{2}+16 i P_{0} \gamma+8 i \lambda \gamma^{2}-4 K^{2} \lambda \gamma+4 \lambda^{2} \gamma^{2}-G^{2} \Gamma^{4}-12 \lambda^{2} P_{0}^{2}-8 K^{2} P_{0}\right. \\
& +12 P_{0}^{2}+\alpha^{2} \Gamma^{4}+8 i \lambda P_{0} \alpha \Gamma^{2}+4 i \lambda \gamma G \Gamma^{2}+2 K^{2} \alpha \Gamma^{2}+32 P_{0} \lambda \gamma+8 \lambda P_{0} G \Gamma^{2}+8 i \lambda P_{0} K^{2}-2 K^{2} i G \Gamma^{2}+8 P_{0} i G \Gamma^{2} \\
& \left.-2 i \alpha \Gamma^{4} G-16 i \lambda^{2} P_{0} \gamma-4 i \alpha \Gamma^{2} \gamma-4 \alpha \Gamma^{2} \lambda \gamma-4 \gamma^{2}+K^{4}\right)^{\frac{1}{2}}<0
\end{aligned}
$$

in the same way if $(\lambda, \gamma, G \neq 0)$ it remains invalid. To analyze the MI gain spectrum, we suppose that $\lambda=\gamma=G=0$. Consequently the increase rate of MI gain spectrum $G(K)=2 \operatorname{Im}(K)$ is given by

$$
G(K)=\sqrt{-8 \alpha P_{0} \Gamma^{2}-8 K^{2} P_{0}+12 P_{0}^{2}+\alpha^{2} \Gamma^{4}+2 \alpha K^{2} \Gamma^{2}+K^{4}}
$$

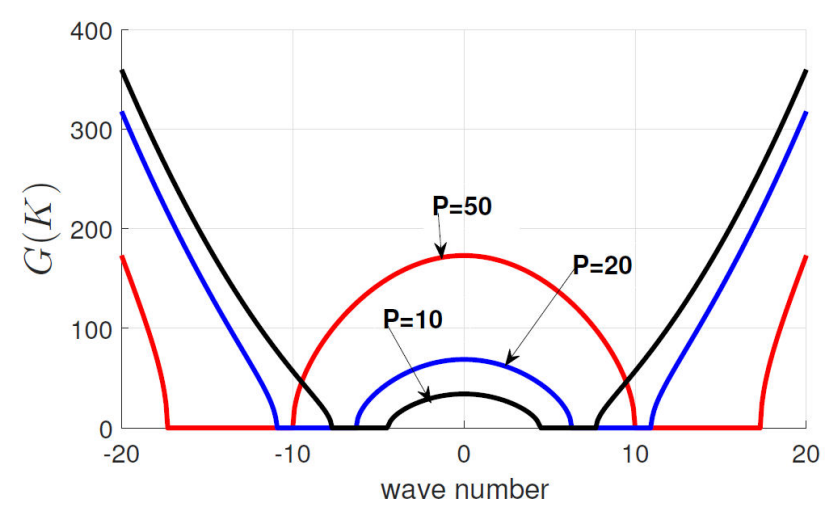

FIGURE 2. Plot of the MI gain spectrum versus wave number with the effect of the incident power (black line $\left[P_{0}=10\right]$, blue line $[P=50]$ and red line $[P=20])$ at $\alpha=0.75, \Gamma=0.75$.

\subsection{Physical explanation and Modulation instability analysis}

Figure 1 is the illustration of the MI gain spectrum versus wave number with the effect of incident power. It is observed that when the incident power increase the unstable band also increase. So, at the maximum incident power, it remains stable (see Fig. 1d). Meanwhile, Fig. 2 is illustration of the MI gain spectrum with small value of the incident power. The unstable plage increase when the incident power value is to small (see black line). Futhermore, Fig. 3 is the illustration of the MI gain spectra versus wave number under the effect of the transverse wave number. For $\Gamma=70.50$, one side lobe is obtained and $38 \leq K \leq 100$. Figures $4 \mathrm{a}$ ) and b) have depicted the chirped bright $3 \mathrm{D}$ and $2 \mathrm{D}$ for values of $n=0.5$, $m=0.14$,
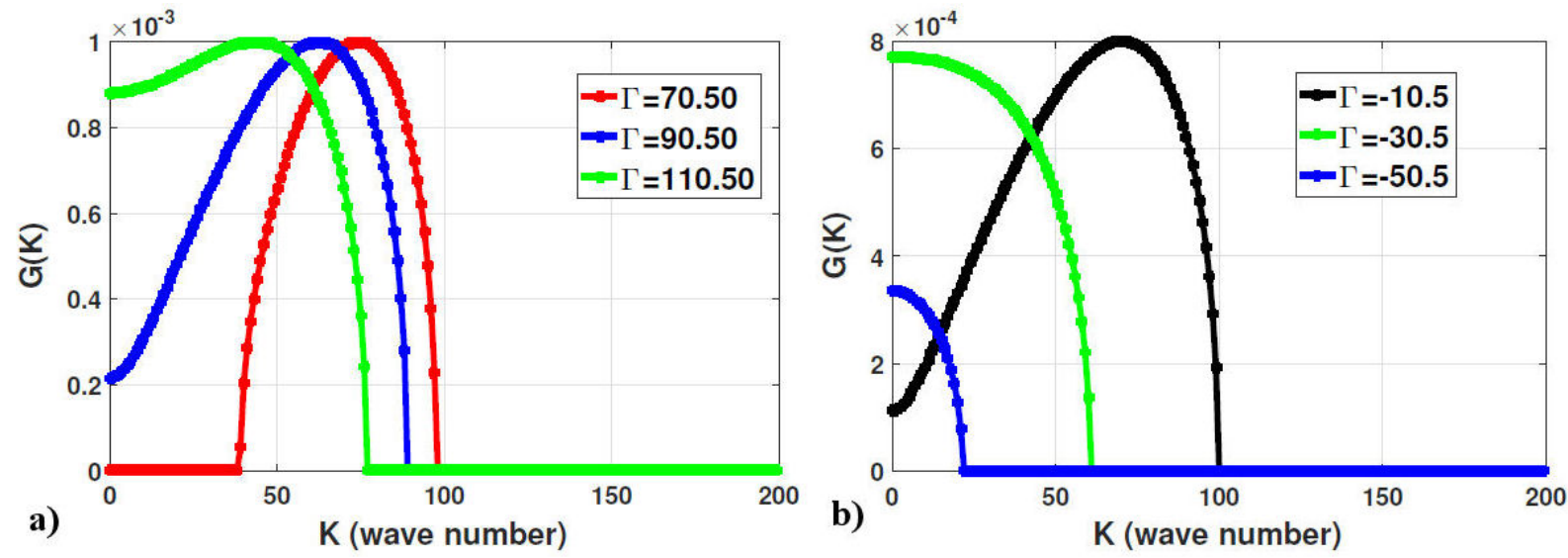

FIGURE 3. (Color online) variation of the MI gain spectra versus wave number with the effect of the transverse wave number a) $[\Gamma=$ $70.50, \Gamma=90.50, \Gamma=110.50]$ and b) $[\Gamma=-10.50, \Gamma=-30.50, \Gamma=-50.50]$ at $\alpha=0.05, P_{0}=2500$ respectively. 


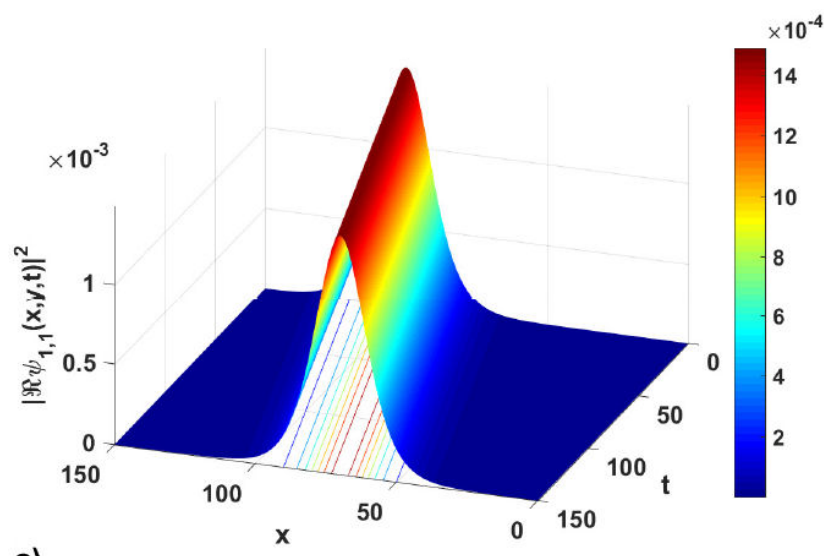

a)

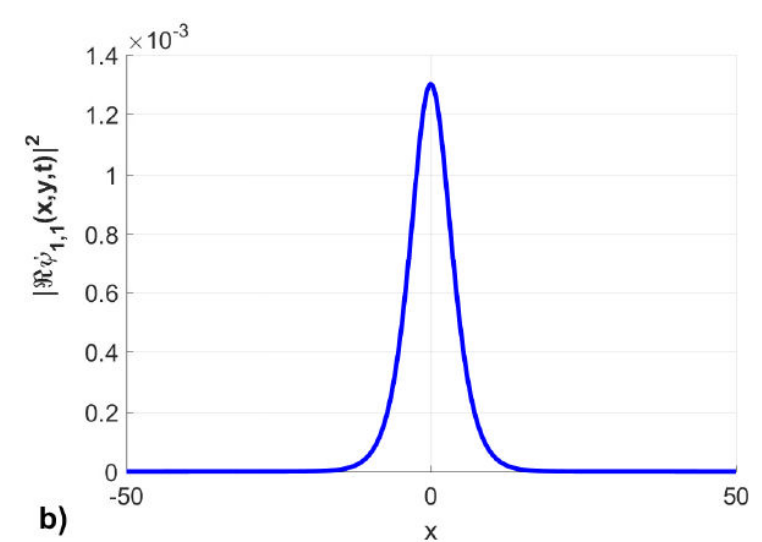

FIGURE 4. Spatiotemporal plot evolution a) 3-D (left panel) and b) 2-D (right panel) of the chirp bright of $\left|\Re \psi_{1,1}(x, y, t)\right|^{2}$ of Eq. (16) at $n=0.5, m=0.14, \alpha=0.02, G=2.7, l=-0.3, \gamma=0.12, y=0.5, E=-0.5, C=0.1106, \omega=0.15$.
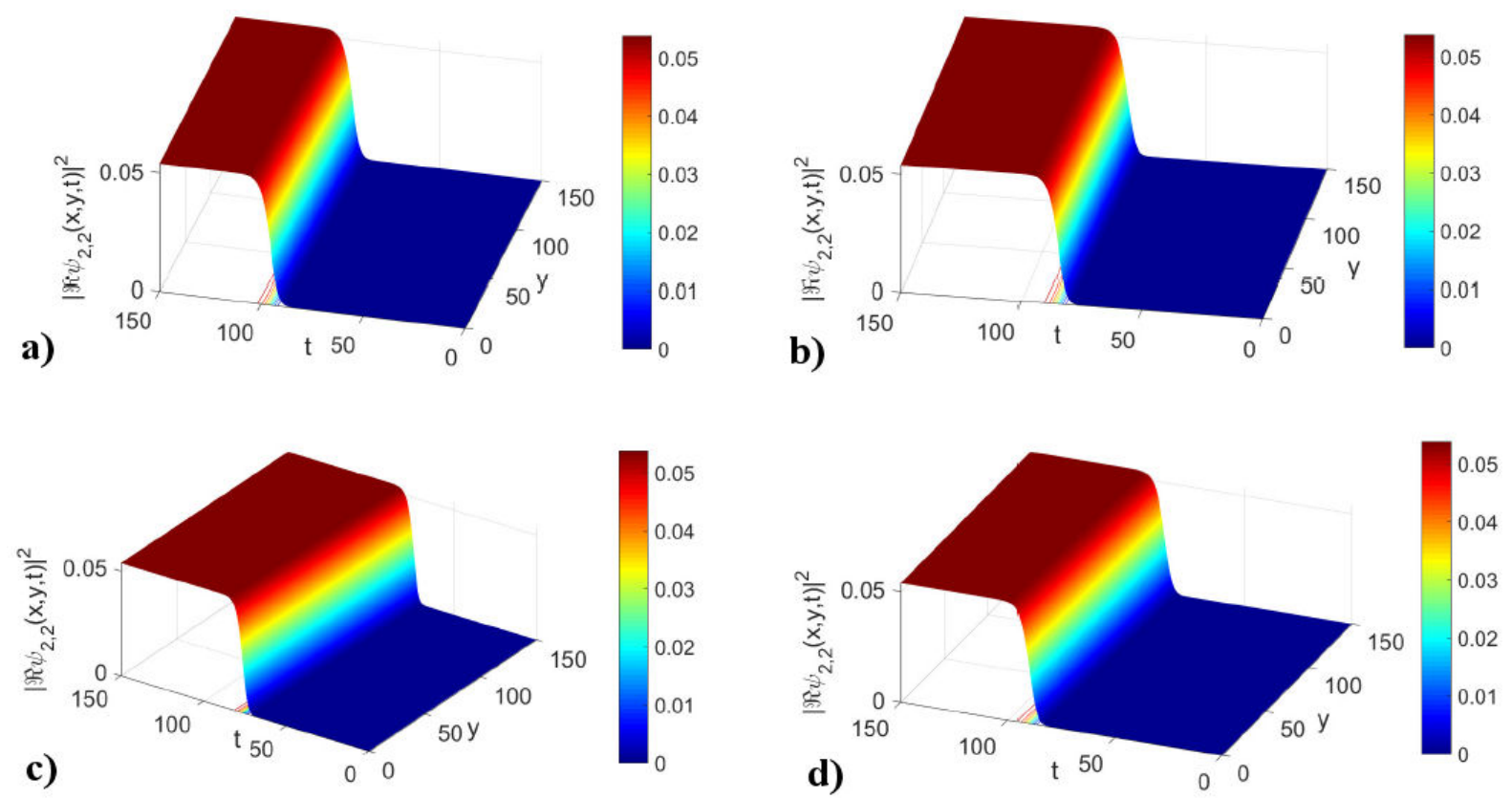

FIGURE 5. Spatiotemporal plot evolution 3-D of the chirp kink-like soliton solutions of $\left|\Re \psi_{2,2}(x, y, t)\right|^{2}$ of Eq. (24) at a) $\omega=2.05$, b) $\omega=2.005$, c) $\omega=2.0005$, d) $\omega=2.00005$, for $A_{0}=-7.5, n=0.5, m=0.14, \alpha=0.2, G=1.7, l=-1.3, \gamma=0.12, x=0$, $E=1.5, C=0.3820$.

$\alpha=0.02, G=2.7, l=-0.3, \gamma=0.12, y=0.5$, $E=-0.5, C=0.1106, \omega=0.15$. Moreover, Figs. 5a), b), c) and d) are chirp kink-like soliton obtained for the values of $A_{0}=-7.5, n=0.5, m=0.14, \alpha=0.2, G=1.7, l=$ $-1.3, \gamma=0.12, x=0, E=1.5, C=0.3820$.

\section{Conclusion}

This work addresses new shape of the chirped bright and dark soliton solutions through the CGLE by using the new sub-ODE equations. By using a special ansatz of the traveling wave transformation, we obtain a new shape of the chirp comparatively to the previous works reported in literature [26,31-34,36,45]. In addition, new singular soliton solutions, trigonometric function solutions and complex traveling waves have been obtained. The obtained results are new in the field of solitons. The authors hope that these results will be very useful to explain physical phenomenons in diverse field of science and engineering. In addition, the different parameters of the CGLE have play an important role during graphical representation of the analytical results. Finally, the linear analysis technique has been applied to the investigation the steady state of the MI gain spectrum and also to point out the different regime of instability. 
1. M. A. Ablowitz and P. A. Clarkson, Solitons, Nonlinear Evolution Equations and Inverse Scattering (Cambridge University Press, Cambridge, 1991), https://doi.org/10.1017/ CB09780511623998.

2. S. Nestor et al., New solitary waves for the Klein-GordonZakharov equations, Mod. Phys. Lett. B 34 (2020) 2050246, https://doi.org/10.1142/S0217984920502462

3. Z. Korpinar, M. Inc, M. Bayram, and M. S. Hashemi, New optical solitons for Biswas-Arshed equation with higher order dispersions and full nonlinearity, Optik 206 (2020) 163332, https://doi.org/10.1016/j.ijleo. 2019.163332

4. R. Fedele, H. Schamel, V. I. Karpman, and P. K. Shukla, Envelope solitons of nonlinear Schrödinger equation with an anti-cubic nonlinearity, J. Phys. A 36 (2003) 1169, https: //doi.org/10.1088/0305-4470/36/4/322,

5. A. Ali, A. R. Seadawy, and D. Lu, Soliton solutions of the nonlinear Schrödinger equation with the dual power law nonlinearity and resonant nonlinear Schrödinger equation and their modulation instability analysis, Optik 145 (2017) 79, https : //doi.org/10.1016/j.ijleo.2017.07.016.

6. E. M. E. Zayed and K. A. E. Alurrfi, New extended auxiliary equation method and its applications to nonlinear Schrödingertype equations, Optik 127 (2016) 9131, https://doi. org/10.1016/j.ijleo.2016.05.100

7. E. C. Aslan and M. Inc, Optical soliton solutions of the NLSE with quadratic- cubic-Hamiltonian perturbations and modulation instability analysis, Optik 196 (2019) 162661, https : //doi.org/10.1016/j.ijleo.2019.04.008.

8. M. Al Ghabshi, E. V. Krishnan, M. Alquran, and K. Al-Khaled, Jacobi elliptic function solutions of a nonlinear Schrödinger equation in metamaterials, Nonlinear Stud. 24 (2017) 469.

9. Z. Korpinar and M. Inc, Numerical simulations for fractional variation of $(1+1)$-dimensional Biswas-Milovic equation, Optik 166 (2018) 77, https://doi.org/10.1016/j. ijleo.2018.02.099

10. X.-F. Yang, Z.-C. Deng, and Y. Wei, A Riccati-Bernoulli subODE method for nonlinear partial differential equations and its application, Adv. Differ. Equ. 2015 (2015) 117, https : //doi.org/10.1186/s13662-015-0452-4

11. A. Biswas, Temporal 1-Soliton Solution of the Complex Ginzburg-Landau Equation with Power Law Nonlinearity, Prog. Electromag. Res. 96 (2009) 1, https://doi.org/ 10.2528/P IER09073108

12. M. Mirzazadeh et al., Optical solitons with complex GinzburgLandau equation, Nonlinear Dyn. 85 (2016) 1979, https: //doi.org/10.1007/s11071-016-2810-5

13. A. H. Arnous, A. R. Seadawy, R. T. Alqahtani, and A. Biswas, Optical solitons with complex Ginzburg-Landau equation by modified simple equation method, Optik 144 (2017) 475, https://doi.org/10.1016/j.ijleo. 2017.07 .013

14. A. Biswas and R. T. Alqahtani, Optical soliton perturbation with complex Ginzburg-Landau equation by semi-inverse variational principle, Optik 147 (2017) 77, https: //doi.org/ $10.1016 / j . i j l e o .2017 .08 .018$
15. H. Triki et al., Bright and dark solitons of the modified complex Ginzburg- Landau equation with parabolic and dual-power law nonlinearity, Rom. Rep. Phys. 64 (2012) 367.

16. A. Biswas et al., Optical soliton perturbation for complex Ginzburg-Landau equation with modified simple equation method, Optik 158 (2018) 399, https://doi.org/10. 1016/j.ijleo.2017.12.131

17. A. Biswas et al., Optical soliton perturbation with complex Ginzburg-Landau equation using trial solution approach, Optik 160 (2018) 44, https://doi.org/10.1016/j. ijleo.2018.01.102

18. A. Biswas, Chirp-free bright optical solitons and conservation laws for complex Ginzburg-Landau equation with three nonlinear forms, Optik 174 (2018) 207, https://doi.org/10. $1016 / j . i j l e 0.2018 .08 .063$

19. S. Arshed, A. Biswas, F. Mallawi, and M. R. Belic, Optical solitons with complex Ginzburg-Landau equation having three nonlinear forms, Phys. Lett. A 383 (2019) 126026, https: //doi.org/10.1016/j.physleta.2019.126026.

20. A. Das et al., Optical solitons with complex Ginzburg-Landau equation for two nonlinear forms using F-expansion, Chin. J. Phys. 61 (2019) 255, https://doi.org/10.1016/j. cjph.2019.08.009

21. Y. Yildirim, A. Biswas, S. Khan, A. S. Alshomrani, and M. R. Belic, Optical solitons with differential group delay for complex Ginzburg-Landau equation having Kerr and parabolic laws of refractive index, Optik 202 (2020) 163737, https: //doi.org/10.1016/j.ijleo.2019.163737

22. E. M. E. Zayed et al., Optical solitons with complex Ginzburg-Landau equation having a plethora of nonlinear forms with a couple of improved integration norms, $O p$ tik 207 (2020) 163804, https://doi.org/10.1016/j. ijleo.2019.163804

23. Y. Yildirim et al., Optical solitons with differential group delay for complex Ginzburg-Landau equation, Res. Phys. 16 (2020) 102888, https://doi.org/10.1016/j.rinp.2019. 102888 .

24. Y. Yan, W. Liu, Q. Zhou, and A. Biswas, Dromionlike structures and periodic wave solutions for variablecoefficients complex cubic-quintic Ginzburg- Landau equation influenced by higher-order effects and nonlinear gain, Nonlinear Dyn. 99 (2020) 1313, https://doi.org/10.1007/ s11071-019-05356-0

25. A. Houwe et al., Complex traveling-wave and solitons solutions to the Klein- Gordon-Zakharov equations, Res. Phys. 17 (2020) 103127, https://doi.org/10.1016/j.rinp.2020. 103127 .

26. A. Houwe, M. Inc, S. Y. Doka, M. A. Akinlar, and D. Baleanu, Chirped solitons in negative index materials generated by Kerr nonlinearity, Res. Phys. 17 (2020) 103097, https://doi. org/10.1016/j.rinp.2020.103097

27. Z. Zhao, Z. Dai, and D. Li, Breather type of chirped soliton solutions for the 2D Ginzburg-Landau equation, Commun. Nonlinear Sci. Numer. Simul. 16 (2011) 1712, https://doi. org/10.1016/j.cnsns.2010.08.026 
28. I. S. Aranson and L. Kramer, The world of the complex Ginzburg-Landau equation, Rev. Mod. Phys. 74 (2002) 99, https://doi.org/10.1103/RevModPhys.74.99

29. Y. Shi, Z. Dai, and D. Li, Application of Exp-function method for 2D cubic- quintic Ginzburg-Landau equation, Appl. Math. Comput. 210 (2009) 269, https://doi.org/10.1016/ j.amc.2009.01.005

30. P. Zhong, R. Yang, and G. Yang, Exact periodic and blow up solutions for 2D Ginzburg-Landau equation, Phys. Lett. A 373 (2008) 19, https://doi.org/10.1016/j. physleta.2008.10.078

31. M. A. Abdou et al., Dark-singular combo optical solitons with fractional complex Ginzburg-Landau equation, $O p$ tik 171 (2018) 463, https://doi.org/10.1016/j. ijleo.2018.06.076

32. G. Akram and N. Mahak, Application of the first integral method for solving $(1+1)$ dimensional cubic-quintic complex Ginzburg-Landau equation, Optik 164 (2018) 210, https: //doi.org/10.1016/j.ijleo.2018.02.108

33. M. S. Osman, On complex wave solutions governed by the 2D Ginzburg- Landau equation with variable coefficients, Optik 156 (2018) 169, https://doi.org/10.1016/j. ijleo.2017.10.127

34. C. Yue, D. Lu, M. Arshad, N. Nasreen, and X. Qian, BrightDark and Multi Solitons Solutions of (3+1)-Dimensional Cubic-Quintic Complex Ginzburg-Landau Dynamical Equation with Applications and Stability, Entropy 22 (2020) 202, https://doi.org/10.3390/e22020202

35. M. Inc and D. Baleanu, Optical solitons for the KunduEckhaus equation with time dependent coefficient, Optik 159 (2018) 324, https://doi.org/10.1016/j.ijleo. 2018.01 .084

36. S. Nestor et al., Diverse chirped optical solitons and new complex traveling waves in nonlinear optical fibers, Commun. Theor. Phys. 72 (2020) 065501, https://doi.org/10. 1088/1572-9494/ab7ecd.

37. S. Nestor, A. Houwe, G. Betchewe, M. Inc, and S. Y. Doka, A series of abundant new optical solitons to the conformable space-time fractional perturbed nonlinear Schrödinger equation, Phys. Scr. 95 (2020) 085108, https : / / doi.org/10. 1088/1402-4896/ab9dad

38. A. I. Aliyu, M. Inc, A. Yusuf, and D. Baleanu, Optical solitons and stability analysis with spatio-temporal dispersion in Kerr and quadric-cubic nonlinear media, $O p$ tik 178 (2019) 923, https://doi.org/10.1016/j. ijleo.2018.10.046

39. A. Korkmaz, O. E. Hepson, K. Hosseini, H. Rezazadeh, and M. Eslami, Sine- Gordon expansion method for exact solutions to conformable time fractional equations in RLW-class, J. King Saud Univ. Sci. 32 (2020) 567, https : / / doi .org/ $10.1016 / j . j k s u s .2018 .08 .013$

40. D. Kumar, K. Hosseini, F. Samadani, The sine-Gordon expansion method to look for the traveling wave solutions of the Tzitzéica type equations in nonlinear optics, $O p$ tik 149 (2017) 439, https://doi.org/10.1016/j. ijleo.2017.09.066

41. C. Yan, A simple transformation fo nonlinear waves, Phys. Lett. A 224 (1996) 77, https://doi.org/10.1016/ S0375-9601 (96)00770-0

42. M. Inc, A. I. Aliyu, A. Yusuf, and D. Baleanu, Optical solitons to the resonance nonlinear Schrödinger equation by Sine-Gordon equation method, Superlattices Microstruct. 113 (2018) 541,

43. K. Khan and M. Ali Akbar, Application of $\operatorname{Exp}(-\phi(\xi))$ expansion Method to Find the Exact Solutions of Modified Benjamin-Bona-Mahony Equation, World Appl. Sci. J. 24 (2013) 1373.

44. A. Houwe et al., Solitons solutions of nonlinear Schrödinger equation in the left-handed metamaterials by three different techniques, J. Phys. Commun. 3 (2019) 011002, https: // doi.org/10.1088/2399-6528/aaff2c

45. A. Houwe et al., Chirped solitons in discrete electrical transmission line, Res. Phys. 18 (2020) 103188, https://doi. org/10.1016/j.rinp.2020.103188 